\title{
Bisphosphonate-Associated Osteonecrosis
}

National Cancer Institute

\section{Source}

National Cancer Institute. Bisphosphonate-Associated Osteonecrosis. NCI Thesaurus.

Code C94497.

Necrosis of the mandible or maxilla in patients receiving intravenous or oral

bisphosphonate therapy. It usually manifests with slow healing or failure to heal of a jaw bone following oral surgery. In a minority of cases, the necrosis appears spontaneously. Signs and symptoms include pain, swelling and infection of the affected area. 